\title{
ATHEROPROTECTIVE EFFECT OF SOLANUM BETACEUM ON RAT EXPOSED TO CIGARETTE SMOKE
}

\section{SITI KHAERUNNISA ${ }^{1 *}$, HANIK BADRIYAH HIDAYATI², JONI SUSANTO ${ }^{3}$, YUANI SETIAWATI ${ }^{4}$, SUHARTATI SUHARTATI ${ }^{5}$}

${ }^{1}$ Departement of Medical Biochemistry, Faculty of Medicine, Airlangga University, Surabaya, Indonesia. ${ }^{2}$ Departement of Neurology, Airlangga University, Dr. Soetomo Hospital, Surabaya, Indonesia. ${ }^{3}$ Departement of Anatomy and Histology, Faculty of Medicine, Airlangga University, Surabaya, Indonesia. ${ }^{4}$ Departement of Pharmacology, Faculty of Medicine, Airlangga University, Surabaya, Indonesia. ${ }^{5}$ Departement of Medical Biochemistry, Faculty of Medicine, Wijaya Kusuma University, Surabaya, Indonesia. Email: st.khaerunnisa@fk.unair.ac.id

Received: 19 December 2018, Revised and Accepted: 17 July 2019

ABSTRACT

Objective: The objective of this research was to determine the atheroprotective effect of Solanum betaceum ethanol extract toward carotid artery intima-media thickness (cIMT) and the level of malondialdehyde (MDA) on rats exposed to cigarette smoke.

Methods: Thirty adult Rattus norvegicus strain Wistar were divided into five groups and exposed to cigarette smoke, 3 pc cigarette/day and simultaneously administered with S. betaceum in Group K2, K3, and K4 (100 mg/kg b.w/day; 200 mg/kg b.w/day; and 400 mg/kg b.w/day), respectively. The duration of treatment for all groups was 28 days. Blood was withdrawn from the cardiac to determine the MDA level. Histological slide from carotid artery intima-media was collected to determine cIMT.

Results: $S$. betaceum ethanol extract administration could significantly prevent the development of atherosclerosis due to oxidative stress by decreasing the level of MDA $(\mathrm{p}<0.05)$ and reducing the degree of cIMT changes $(\mathrm{p}<0.05)$.

Conclusion: The present study found that $S$. betaceum ethanol extract could prevent the development of atherosclerosis due to smoke exposure through the reduction of the MDA level, i.e., the marker of oxidative stress, which is associated with the reduced of cIMT changes. However, further studies on other bioactivity of $S$. betaceum as an antioxidant are warranted.

Keywords: Solanum betaceum, Malondialdehyde, cIMT, Atherosclerosis, Smoke exposure.

(C) 2019 The Authors. Published by Innovare Academic Sciences Pvt Ltd. This is an open access article under the CC BY license (http://creativecommons. org/licenses/by/4. 0/) DOI: http://dx.doi.org/10.22159/ijap.2019.v11s5.T1005

\section{INTRODUCTION}

Cardiovascular disease (CVD) is the primary cause of death in both developed and developing country. Coronary artery disease occurs in as much $42 \%$ of males and $25 \%$ of females, with an even higher percentage in people with other complications. It is estimated that until 2030, as much as 23.3 million people will die due to CVD [1,2]. One of the main causes of this disease is oxidative stress, which is an imbalance between oxidants and antioxidants inside the body [3]. Evidence shows that oxidative stress plays a significant role in the pathogenesis and development of CVD, among them being atherosclerosis [3,4].

Atherosclerosis is a systemic disease responsible for most incidences of CVD s and stroke [5]. Atherosclerosis is a complex phenomenon that is the main cause of coronary artery disease, which can be illustrated an excess of fibro-fatty substance, or the accumulation of atheroma plaques, is an inflammatory response toward arterial wall damage $[6,7]$. Exposure to cigarette smoke is one of the main risk factors toward the early development of atherosclerosis and other CVD complications. Several main components of cigarette smoke are nicotine, tar, and carbon monoxide. These substances also have a negative influence on the body, both for the respiratory and the cardiovascular system [8]. Cigarette smoke also contains some free radicals. These free radicals can initiate the development of reactive oxygen species (ROS), which are reactive molecules that are constantly formed by the enzymatic reactions within the body $[9,10]$. When the level of ROS increases past that of the body's antioxidant defense system, oxidative stress occurs $[9,10]$. Oxidative stress creates substances that can cause cytotoxicity in the endothelial cells and smooth muscles of the blood vessel, which includes several variations of aldehyde, one of which is malondialdehyde (MDA) [11,12].

Oxidative stress is reported to have a significant role in the initiation and development of atherosclerosis by stimulating the production of inflammatory factors and cytokines [13]. Atherosclerosis is characterized by the weakening of the vessel walls, inflammatory response, the formation of foam cell plaques, and the formation of blood clots. The oxidative stress process causes endothelial dysfunction, and on the other hand, endothelial dysfunction increases the state of constant oxidative stress, thus causing the development of atherosclerosis [4].

This alteration of the endothelial function plays an important role in the pathophysiology of atherosclerosis [14]. A published research on the topic has managed to prove the presence of foam cells in the blood vessels, from the tunica intima to the tunica media in the aorta of rats that have been exposed to cigarette smoke for 20-30 days [15]. The early phase of atherosclerosis is alteration response of medial cells that can be assessed by carotid artery intima-media thickness (cIMT) measurement, and later stages are the generation of carotid plaques [14]. Measurement of the intima-media thickness (IMT) of the common carotid artery is an early morphological marker for the occurrence of atherosclerosis [5]. IMT is an indicator of atherosclerosis risk factors and the treatment given to reduce the influence of those risk factors $[11,14,16]$.

The consumption of vegetable-based food is also linked to a low risk of CVD and hypertension. These last decades, several researches have 
focused on secondary metabolic substances of plants, for example, flavonoid, as a substance that may help prevent CVD s $[17,18]$. Tamarillo (Solanum betaceum), which is also known in Indonesian as Terong belanda is a plant originating from the Solanaceae family. A majority of our citizens are not familiar with Tamarillo, often preferring eggplants as their daily vegetable of choice. Prior researches have shown that $S$. betaceum has a neuroprotective effect. The phytochemical component reported to be present in $S$. betaceum includes flavonoid, tannin, and terpenoid [19].

This research was done to analyze the atheroprotective effects of $S$. betaceum ethanol extract, measured through observed histopathological changes of the cIMT and oxidative stress parameters measured through the presence of MDA, as a possible early preventative measure against CVD due to cigarette smoke exposure.

\section{METHODS}

\section{Experimental design}

The present study was carried out in accordance with the guidelines of Ethical Clearance provided by the Animal Care and Use Committee, Veterinary Faculty of Airlangga University with certificate number 742-KE. This experimental study used Rattus norvegicus strain Wistar ( 3 months old, $\pm 180 \mathrm{~g}$ ) in a healthy condition. The animals were divided into five groups and acclimatized to the laboratory conditions and maintained under $12 \mathrm{~h}$ light and dark cycles at room temperature. The animals received a standard diet and water ad libitum: $\mathrm{K}_{0}$ : Negative control group, $\mathrm{K}_{1}$ : Positive control group (smoke exposure), $\mathrm{K}_{2}$ : Treatment Group 1 (smoke exposure and ethanol extract of $S$. betaceum $100 \mathrm{mg} / \mathrm{BW}$ ) [20], $\mathrm{K}_{3}$ : Treatment Group 2 (smoke exposure and ethanol extract of $S$. betaceum $200 \mathrm{mg} / \mathrm{kgBW}$ ) [20], and $\mathrm{K}_{4}$ : Treatment Group 3 (smoke exposure and ethanol extract of S. betaceum $400 \mathrm{mg} / \mathrm{kgBW}$ ) [20].

\section{The measurement of carotid artery IMT (cIMT)}

Carotid artery tissue that has been isolated is fixated with formalin $10 \%$. It is then made into a histologic slide and treated with Hematoxylin Eosin (H\&E) coloring. Observation is done with an Olympus microscope, with $\times 100$. The IMT is evaluated by measuring the tunica intima and media from the innermost endothelial boundary to the outermost tunica media using the cellSens software.

The measurement of malondialdehyde levels (MDA)

MDA level is obtained from the serum of $R$. norvegicus rats, Wistar strain, and is calculated with the thiobarbituric acid reactive substance method, and observed with a spectrophotometer at a wavelength of $529 \mathrm{~nm}$, measured in $\mathrm{nmol} / \mathrm{ml}$ [21]. Level of MDA was obtained from Zeptometrix, Buffalo, New York, USA, with Cat No.0801192.

\section{The preparation of $S$. betaceum ethanol extract}

$S$. betaceum was purchased from farmland in Wonosobo, Central Java, Indonesia, and identified from Indonesian Institute of Science. $S$. betaceum was dried by fresh dryer. Dry powder was extracted by maceration using ethanol solvent for $3 \times 24 \mathrm{~h} 3$ times at room temperature. The ethanol extract of $S$. betaceum was added to the treatment diet in the form of a suspension using 1\% CMC with a dose of $2 \mathrm{ml} / 200 \mathrm{~g}$ b.w. $S$. betaceum extract was simultaneously administered to the treatment group before exposure to cigarette smoke during the experiment.

\section{Cigarette smoke exposure}

Exposure of cigarette smoke in experimental animals was given to groups $\mathrm{K} 1, \mathrm{~K} 2, \mathrm{~K} 3$, and $\mathrm{K} 4$ by 3 pc cigarettes per day for 28 days, at 6.00 p.m. The exposure system consisted of a glass box with two holes; several holes to insert cigarette smoke and other holes to remove cigarette smoke. A 50 cc syringe is pulled out to suck the cigarette placed on the iron pipe, so the cigarette smoke entered the syringe. The process of pumping $50 \mathrm{cc}$ syringe is done repeatedly until the cigarette burned out. Locally available brand of cigarette, manufactured in Surabaya, East Java, Indonesia, was used in this study.

\section{Statistical analysis}

The statistical analysis was performed by one-way ANOVA and KruskalWallis test. The statistical significance between groups was assessed by the least significant difference (LSD) and Mann-Whitney U-test with $\mathrm{p}<0.05$. Data analysis was used by SPSS ver.23.

\section{RESULTS AND DISCUSSION}

The average result of cIMT measurement in control and experimental animals are summarized in Table 1 and Fig. 1a. A significant increase $(\mathrm{p}<0.05)$ in cIMT was observed in Group K1 (rats exposed to cigarette smoke) when compared to Group K0 (control group rats). S. betaceumadministered rats (Group K2, K3, and K4) showed a significant decrease in their cIMT $(\mathrm{p}<0.05)$ compared to Group $\mathrm{K} 1$ rats, which shows the atheroprotective effect of the substance against cigarette smoke exposure.

The level of MDA was observed by TBARC method from the serum. The level of MDA in the different groups is shown in Table 2 and Fig. 1 b. A significant increase $(\mathrm{p}<0.05)$ in MDA levels was observed in Group K1 (rats exposed to cigarette smoke) when compared to rats belonging to

Table 1: Mean, standard deviation, and ANOVA test of carotid artery intima-media thickness of rats control and treatment groups eme

\begin{tabular}{lllllll}
\hline Variable & Group $^{\#}$ & & & & \\
& \cline { 2 - 6 } & K0 & K1 & K2 & ANOVA \\
cIMT & $32.76 \pm 5.06^{\mathrm{a}}$ & $46.77 \pm 5.41^{\mathrm{b}, \mathrm{d}}$ & $38.18 \pm 5.17^{\mathrm{a}, \mathrm{c}}$ & $40.09 \pm 2.43^{\mathrm{a}, \mathrm{d}}$ & $41.05 \pm 10.58^{\mathrm{c}, \mathrm{d}}$ & $\mu$ Km $^{0.024^{*}}$ \\
\hline
\end{tabular}

*Significantly with $\mathrm{p}<0.05$, "Mean \pm SD, a,b,c,d Different superscript means significant between groups. cIMT: Carotid artery intima-media thickness, SD: Standard deviation, SI: System International of unit

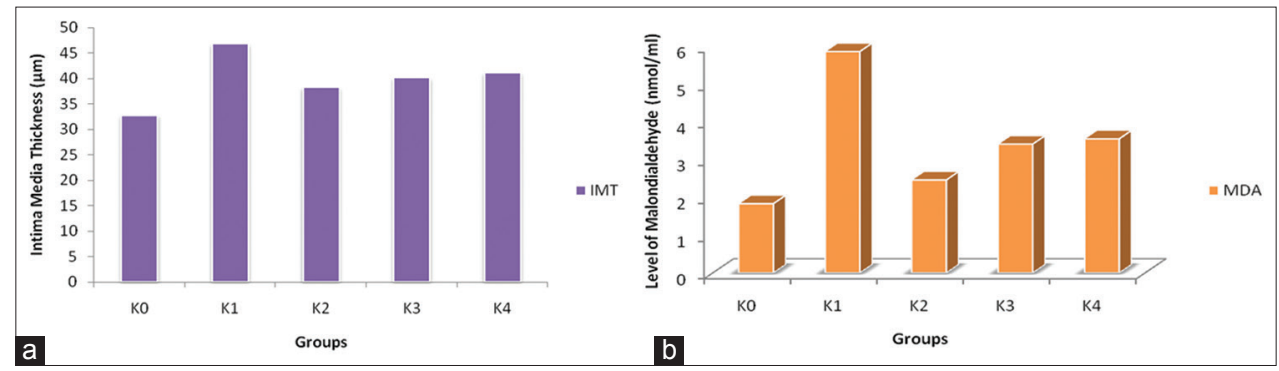

Fig. 1: (a) Average degree of cIMT among experimental groups, (b) level of malondialdehyde among experimental groups. K0 = negative control group, $\mathrm{K} 1$ = positive control group, K2 = treatment Group 1, K3 = treatment Group 2, K4 = treatment Group 3 
Table 2: Mean, median, minimum, maximum, and Kruskal-Wallis test of malondialdehyde of rats control and treatment groups

\begin{tabular}{lllllllll}
\hline Variable & Categori & \multicolumn{2}{l}{ Group } & & & & SI & Kruskal-Wallis \\
\cline { 3 - 7 } & & K0 & K1 & K2 & K3 & K4 & & \\
\hline MDA & Mean & $1.83^{\mathrm{a}}$ & $5.86^{\mathrm{b}}$ & $2.46^{\mathrm{c}}$ & $3.41^{\mathrm{b}, \mathrm{c}}$ & $3.55^{\mathrm{c}}$ & $\mathrm{nmol} / \mathrm{ml}$ & $0.002^{*}$ \\
& Median & 1.80 & 6.86 & 2.33 & 2.96 & 2.33 & & \\
& Minimum & 1.52 & 2.78 & 2.09 & 1.98 & 1.98 & & \\
& Maximum & 2.21 & 8.06 & 3.13 & 5.31 & 6.45 & & \\
\hline
\end{tabular}

*Significantly with $\mathrm{p}<0.05$, a,b,c Different superscript means significant between groups. MDA: Malondialdehyde, SI: System International of unit

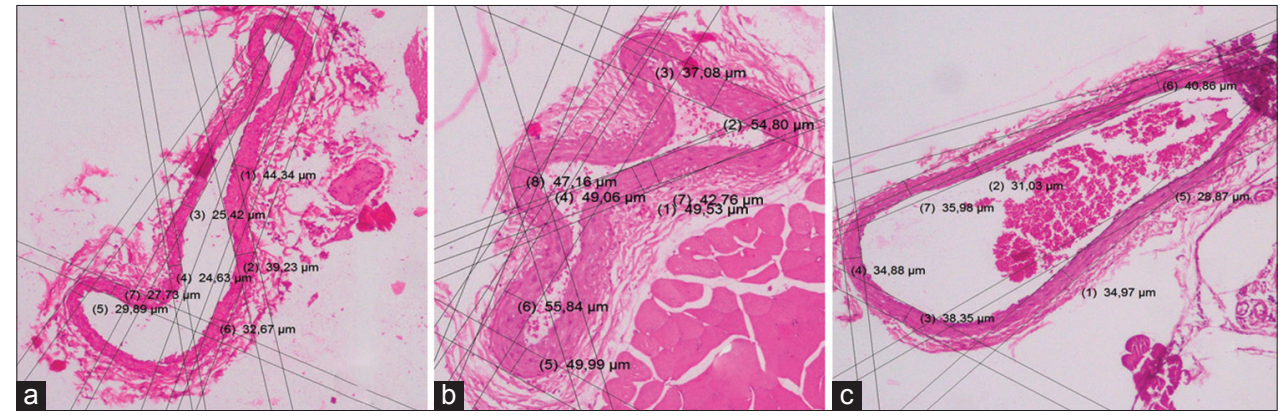

Fig. 2: Histochemistry of carotid artery intima-media thickness

Group K0. Rats belonging to $S$. betaceum - administered groups (i.e., K2, $\mathrm{K} 3$, and K4), exhibited a decrease in their MDA levels when compared to Group K1. This shows the antioxidant effect of $S$. betaceum against cigarette smoke exposure.

The histochemistry of the carotid artery sample was used to evaluate cIMT. The IMT of the common carotid artery is an early morphological marker for the occurrence of atherosclerosis. The research shows that exposure to cigarette smoke alters the cIMT significantly (Fig. 2b) when compared to the normal group (Fig. 2a). The administration of $S$. betaceum ethanol extract could prevent smoke derived cIMT alteration (Fig. 2c), which is significantly compared with positive control group (Fig. 2b) not significantly different when compared to the normal group (Fig. 2a).

The present study disclosed that administration of $S$. betaceum ethanol extract on rats exposed to cigarette smoke could prevent the development of atherosclerosis by reducing the level of MDA and degree of cIMT changes in the samples. Phytochemistry screening analysis in previous studies [19] found that the ethanol extract of $S$. betaceum contains flavonoid, tannin, gallic tannin, and terpenoid which suggested has an important role as atheroprotective in the present study.

\section{Pathogenesis of atherosclerosis (based on oxidative stress study)} due to smoke exposure

The previous study has indicated that active and passive cigarette smoking is associated with dysfunction of endothelial cell [22]. Cigarette smoke contains some free radicals that these can initiate the generation of ROS, which are reactive molecules that are constantly formed by the enzymatic reactions within the body $[9,10]$. ROS compounds are produced by several oxidase enzymes, including nicotinamide adenine dinucleotide phosphate oxidase, xanthine oxidase, uncoupled endothelial nitric oxide synthase (eNOS), cyclooxygenase (COX), glucose oxidation, lipoxygenase, and mitochondrial electron transport [23]. A variety of ROS and reactive nitrogen species (RNS) has been found to be involved in the disease pathology mechanism including radical superoxides $\left(\mathrm{O}^{-}{ }_{2}\right)$, radical hydroxyl $(\mathrm{OH})$, singlet oxygen $\left({ }^{1} \mathrm{O}_{2}\right)$, hydrogen peroxide $\left(\mathrm{H}_{2} \mathrm{O}_{2}\right)$, radical hypochlorite (CLO), heme ferryl species protein, alkoxyl lipid (RO), and radical peroxyl (ROO), radical peroxyl nitrite $\left(\mathrm{ONOO}^{-}\right)$, nitrite oxide (NO), and radical nitrogen dioxide (NOO-) [4,24-26].

This imbalance between oxidants and antioxidants inside the human body has the potential to cause damage through a process known as oxidative stress. It is a cellular, organic, or organism-wide metabolic condition that is characterized by an oxidative excess. The means through which ROS disturbs cellular function cannot be wholly explained as of yet; however, one of the most important mechanisms is the occurrence of lipid peroxidation, which causes cell death [24,27]. One of the products of lipid membrane degradation is MDA, which is produced by the process of lipid peroxidation. This marker has often been identified in patients with atherosclerosis, certain types of cancers, neurodegenerative diseases, and lung illnesses, especially those due to inflammation [24].

Some existing evidence points to the fact that oxidative stress plays a significant role in the pathogenesis and development of CVDs, which includes atherosclerosis [4]. Atherosclerosis is an inflammation of the blood vessel due to the accumulation of atheromatous plaques within the walls of the artery causes the dysfunction of the vessel wall's endothelial cells [18]. Oxidative stress causes endothelial dysfunction and endothelial dysfunction causes constant oxidative stress. This forms a vicious cycle that eventually leads to the formation of atherosclerosis. Oxidative stress is related to a lack of tetrahydrobiopterin and a decrease in eNOS activity, which may lead to endothelial dysfunction in atherosclerotic patients by causing an increase in the oxidative stress process within the body [4].

In patients with high cardiovascular risklevel, the decrease in endothelial NO bioavailability can cause the following conditions: (a) Reduced eNOS expression; (b) a disruption of cellular signals which causes an eNOS substrate or cofactor deficiency and the reduction of eNOs activation; (c) decrease in the endothelial cell's capacity to synthesize and/or release NO; and (d) decrease of NO synthesis by ROS. All these abnormalities may lead to endothelial dysfunction, which is commonly seen as an initial step of the pathogenesis of atherosclerosis [4].

The early phase of atherosclerosis that can be assessed by carotid IMT (cIMT) measurement and later stages are mostly generation of carotid plaques. Increasing of cIMT is typically seen in a common carotid artery due to its flow dynamics, whereas plaques are particularly seen in the internal carotid artery or carotid bulbs. As found in the present studies, cIMT elevation is most often related vascular disorder due to smoke exposure $[14,28]$

\section{Flavonoid; its role as an atheroprotective substance}

The process of atherosclerosis has long been seen as a systemic chronic inflammatory disease; mainly causing morbidity and mortality through 
its consequent cardiovascular illnesses [17]. A high-intake of flavonoids from fruits and vegetables has a known correlation with a lower risk of cardiovascular illnesses. The mechanism to explain the related process is still unclear, but current evidence clearly shows that flavonoid can help reduce cardiovascular risk-factors [18].

The flavonoid, included within the phenolic compound group, is often found in plant tissues and can act as an antioxidant. Flavonoid's antioxidant activity stems from its ability to donate hydrogen atoms or from its ability to chelate metal. Multiple research has shown that flavonoid has anti-allergic, anti-inflammatory, antimicrobial, anticancer, antidiarrhea, and antiviral properties [3,24,29,30]. Several studies have proven that a high intake of flavonoid is positively linked with a significant reduction of coronary heart disease risk. The vascular endothelial cells play a significant role in safeguarding the health of the heart by producing nitric oxide, a substance that relaxes arteries (causes vasodilatation). The production of endothelial nitric oxide can inhibit platelet adhesion and aggregation, which is one of the preliminary factors in the formation of a blood clot. A variety of clinical studies have researched the potential of high flavonoid intake in reducing a multitude of platelet aggregation sizes; these trials have reported different results. Food-based flavonoids were reported to potentially be involved in cardiovascular-disease prevention, mainly by reducing oxidative stress and improving NO bioavailability. Flavonoid is to modulate genes that are related to metabolism, stress-defense, enzyme metabolism, detoxification, and protein transporters [18].

A majority of flavonoids work as an effective free-radical scavenger. However, this effect may not always be beneficial because flavonoids turn into radical flavonoids after they complete their scavenging functions. However, some radical flavonoids with high-stability will not easily react and will instead act as an antioxidant. The position and total number of hydroxyl chains, its configuration, substitution, and other factors in the flavonoid chemical structure contribute to its ability to chelate metallic ion, which in turns helps them scavenge and inhibit free radicals $[3,21,23]$. The hydroxyl chain configuration in the B-ring significantly muffles (scavenges) and chelates metallic ion from ROS and RNS due to their function as hydrogen and electron donor to hydroxyl, peroxyl, and radical peroxynitrite chains, which produces relatively stable radical flavonoids or radical aroxyl (Fl-0) [24,29].

Aside from that, flavonoid also has an antioxidant effect that can improve the length of a cell's life, as well as induce apoptosis and inhibit cell proliferation, which is why it can also act as an anticarcinogenic factor. In line with this information, it is reported that flavonoids such as epigallocatechin-gallate and resveratrol can inhibit transcription factors such as NFkB and AP-1 through an interaction between upstream signaling pathways (IKK phosphorylation, MAPK phosphorylation, and P13K/Akt phosphorylation) and/or by reducing proinflammatory mediators (tumor necrosis factor-a, interleukin, and prostaglandin E2) and the activity of pro-inflammatory enzymes (COX 2, iNOS) [31].

The modulation of cell-signal pathways by flavonoids can help prevent cardiovascular diseases through the following means: (a) reduced inflammation, (b) reduction of the expression of vascular-cell adhesion molecules, (c) increased endothelial nitric oxide synthase (eNOS) activity and (d) reduced platelet aggregation. Nitric oxide is required to maintain vasodilatation. Nitric oxide disorders are often related to a heightened risk of CVDs; (d) reduced platelet aggregation: Thrombocyte aggregation is one of the first steps in the formation of blood clots. These clots may block the coronary arteries or the brain and cause either a myocardial infarction or a stroke. Dealing with platelet aggregation is an important strategy in the primary and secondary prevention of CVD s [18].

Based on the evidence from the current results, our study suggested that the health-promoting capabilities effects of $S$. betaceum in these animal model of rats exposed by cigarette smoke could be attributed to the atheroprotective effects. As preventive from atherosclerosis, we suggest that $S$. betaceum can direct intercept free radical before any significant oxidation can occur which was indicated by decreasing level of MDA. As an antioxidant, S. betaceum can retard or slow the oxidative processes leading to decrease MDA, i.e., end product of lipid peroxidation. Thus, reduce the incidence and prevent atherosclerosis through a inhibit in carotid artery IMT changes induced by cigarette smoke exposure.

\section{CONCLUSION}

The present study findings showed that exposure to cigarette smoke could increase the level of MDA, i.e., marker of oxidative stress and degree of carotid artery IMT (cIMT) changes in the experimental animal. Ethanol extract of $S$. betaceum administration exhibited a powerful antioxidant potential toward lowering the level of MDA and thus reduce the development of atherosclerosis which shown by decreasing the degree of cIMT changes induced by cigarette smoke exposure. However, further studies on other bioactivity of $S$. betaceum are warranted.

\section{ACKNOWLEDGMENT}

We would like to thank Airlangga University for all the support they have given toward the completion of this research.

\section{CONFLICTS OF INTEREST}

All authors have none to declare.

\section{REFERENCES}

1. Andhuvan G, Ayyappan P, Sahana C, Poovizhi S, Sivasakthi K. Knowledge of modifiable risk factors of heart disease among patients with cardiovascular risk. Asian J Pharm Clin Res 2016;10:99.

2. Thomas S, Rich MW. Epidemiology, pathophysiology, and prognosis of heart failure in the elderly. Heart Fail Clin 2007;3:381-7.

3. Saxena M, Saxena J, Pradhan A. Review article. Int J Pharm Sci Rev 2012;16:130-4.

4. Grassi D, Desideri G, Ferri C. Flavonoids: Antioxidants against atherosclerosis. Nutrients 2010;2:889-902.

5. Coll B, Feinstein SB. Carotid intima-media thickness measurements: Techniques and clinical relevance. CurrAtheroscler Rep 2008;10:444-50.

6. Libby P, Ridker PM, Hansson GK. Progress and challenges in translating the biology of atherosclerosis. Nature 2011;473:317-25.

7. Mahdavi-Roshan M, Zahedmehr A, Mohammad-Zadeh A, Sanati HR, Shakerian F, Firouzi A, et al. Effect of garlic powder tablet on carotid intima-media thickness in patients with coronary artery disease: A preliminary randomized controlled trial. Nutr Health 2013;22:143-55.

8. MiYang J, HyeonCheol J, Lee K, Yim J. The acute effect of smoking a single cigarette on vascular status, $\mathrm{SpO} 2$, and stress level. Med Sci Monit 2014;20:601-7.

9. Valavanidis A, Vlachogianni T, Fiotakis K. Tobacco smoke: Involvement of reactive oxygen species and stable free radicals in mechanisms of oxidative damage, carcinogenesis and synergistic effects with other respirable particles. Int J Environ Res Public Health 2009;6:445-62.

10. Dellinger B, Khachatryan L, Masko S, Lomnicki S. Free radicals in tobacco smoke free radicals in tobacco smoke. Mini Rev Org Chem 2011;8:427-33.

11. Moore KP, Darley-Usmar V, Morrow J, Roberts LJ $2^{\text {nd }}$. Formation of F2-isoprostanes during oxidation of human low-density lipoprotein and plasma by peroxynitrite. Circ Res 1995;77:335-41.

12. Ballinger SW, Patterson C, Yan CN, Doan R, Burow DL, Young CG, et al. Hydrogen peroxide- and peroxynitrite-induced mitochondrial DNA damage and dysfunction in vascular endothelial and smooth muscle cells. Circ Res 2000;86:960-6.

13. Fernández-Robredo P, Rodríguez JA, Sádaba LM, Recalde S, GarcíaLayana A. Egg yolk improves lipid profile, lipid peroxidation and retinal abnormalities in a murine model of genetic hypercholesterolemia. J Nutr Biochem 2008;19:40-8.

14. Barakoti M. Carotid intima-media thickness and coronary artery disease: An Indian perspective. Nepal Heart J 2018;15:9-15.

15. Lapatta N, Loho L, Poppy L. Gambaran histopatologi aorta tikus wistar yang dipapar asap rokok. J e Biomedik 2013;1:1019-22.

16. Halenka M, Vaverkova H, Hutyra M, Karasek D, Slavik L, Novotny D, et al. Detection of early atherosclerosis using the ultrasound parameter of the intima-media thickness of the common carotid artery in families 
with familial combined hyperlipidemia. Int Angiol 2004;23:230-7.

17. McCullough ML, Peterson JJ, Patel R, Jacques PF, Shah R, Dwyer JT. Flavonoid intake and cardiovascular disease mortality in a prospective cohort of US adults. Am J Clin Nutr 2012;95:454-64.

18. Suhartati S, Khaerunnisa S. Flavonoid. Surabaya: Airlangga University Press; 2016.

19. Khaerunnisa S, Kusumastuti K, Aminah N, Mustika A, Suhartati S. Mechanism of Solanum betaceum to prevent memory impairment in cigarette smoke exposed rat. Int J App Pharm, 2019;11:20-4.

20. Kandimalla R, Kalita S, Choudhury B, Kotoky J. Review article a review on anti-diabetic potential of genus Solanum (Solanaceae). J Drug Deliv Ther 2015;5:24-7.

21. Mohamed O, Saidb M, Alib Z, Hanan A, Heba S. Improving effect of dietary oat bran supplementation on oxidative stress induced by hyperlipidemic diet. Researcher 2011;3:1-10.

22. Mazzone P, Tierney W, Hossain M, Puvenna V, Janigro D, Cucullo L. Pathophysiological impact of cigarette smoke exposure on the cerebrovascular system with a focus on the blood-brain barrier: Expanding the awareness of smoking toxicity in an underappreciated area. Int J Environ Res Public Health 2010;7:4111-26.

23. Hertzog DI, Tica OS. Molecular mechanisms underlying the anti- cancerous action of flavonoids. Curr Health Sci J 2012;38:145-9.

24. Patel JM. A review of potential health benefits of flavonoids. Lethbridge Undergrad Res J 2010;3:2-6.

25. Halliwell B. Reactive species and antioxidants. Redox biology is a fundamental theme of aerobic life. Plant Physiol 2006;141:312-22.

26. Stefek M. Natural flavonoids as potential multifunctional agents in prevention of diabetic cataract. Interdiscip Toxicol 2011;4:69-77.

27. Sandhar H, Kumar B, Prasher S, Tiwari P. A review of phytochemistry and pharmacology of flavonoids. Int Pharm Sci 2011;1:25-41

28. Bauer M, Caviezel S, Teynor A, Erbel R, Mahabadi AA, SchmidtTrucksäss A. Carotid intima-media thickness as a biomarker of subclinical atherosclerosis. Swiss Med Wkly 2012;142:w13705.

29. Kumar S, Pandey AK. Chemistry and biological activities of flavonoids: An overview. ScientificWorldJournal 2013;2013:162750

30. Middleton E Jr., Kandaswami C, Theoharides TC. The effects of plant flavonoids on mammalian cells: Implications for inflammation, heart disease, and cancer. Pharmacol Rev 2000;52:673-751.

31. Rezzani R, Porteri E, De Ciuceis C, Bonomini F, Rodella LF, Paiardi S, et al. Effects of melatonin and pycnogenol on small artery structure and function in spontaneously hypertensive rats. Hypertension 2010;55:1373-80. 